\title{
Full Waveform Inversion for Time-Distance Helioseismology
}

\author{
Shravan M. Hanasoge ${ }^{1,2,3}$ \& Jeroen Tromp ${ }^{2,4}$ \\ hanasoge@tifr.res.in
}

\begin{abstract}
Inferring interior properties of the Sun from photospheric measurements of the seismic wavefield constitutes the helioseismic inverse problem. Deviations in seismic measurements (such as wave travel times) from their fiducial values estimated for a given model of the solar interior imply that the model is inaccurate. Contemporary inversions in local helioseismology assume that properties of the solar interior are linearly related to measured travel-time deviations. It is widely known, however, that this assumption is invalid for sunspots and active regions, and likely for supergranular flows as well.

Here, we introduce nonlinear optimization, executed iteratively, as a means of inverting for the sub-surface structure of large-amplitude perturbations. Defining the penalty functional as the $L_{2}$ norm of wave travel-time deviations, we compute the the total misfit gradient of this functional with respect to the relevant model parameters at each iteration around the corresponding model. The model is successively improved using either steepest descent, conjugate gradient, or quasi-Newton limited-memory BFGS. Performing nonlinear iterations requires privileging pixels (such as those in the near-field of the scatterer), a practice not compliant with the standard assumption of translational invariance. Measurements for these inversions, although similar in principle to those used in time-distance helioseismology, require some retooling. For the sake of simplicity in illustrating the method, we consider a 2-D inverse problem with only a sound-speed perturbation.
\end{abstract}

\footnotetext{
${ }^{1}$ Department of Astronomy and Astrophysics, Tata Institute of Fundamental Research, Mumbai 400005, India

${ }^{2}$ Department of Geosciences, Princeton University, Princeton, NJ 08544, USA

${ }^{3}$ Max-Planck-Institut für Sonnensystemforschung, 37191 Katlenburg-Lindau, Germany

${ }^{4}$ Program for Applied and Computational Mathematics, Princeton University, Princeton, NJ 08544, USA
} 
Subject headings: Sun: helioseismology—Sun: interior-Sun: oscillationswaves - hydrodynamics

\section{Introduction}

Imaging the non-axisymmetric interior structure and dynamics of the Sun requires interpreting measurements of the photospheric seismic wavefield (see reviews by, e.g., Gizon \& Birch 2005; Gizon et al. 2010). There exist a number of techniques to process observations of the seismic wavefield; in this article we focus on time-distance helioseismology (Duvall et al. 1993), in which travel times of waves are the primary measurements.

Full waveform inversion is a label for set of techniques widely used in terrestrial and exploration seismology to infer the structure of the highly heterogeneous Earth. "Full waveform" refers to the use of the entire seismic measurement (which in the case of helioseismology is the cross correlation) in the inversion. A waveform can broken up into frequency bands, and every part of the waveform can be characterized by parameters such as phase and amplitude. The full-waveform approach involves assimilating all of these measurements into the inversion in the to maximally leverage seismic data. A number of inversion methods already adopt aspects of this approach (e.g., Švanda et al. 2011; Jackiewicz et al. 2012; Dombroski et al. 2013), strictly assuming however that seismic measurements depend linearly on interior properties. In the present formulation, we compare waveforms solely in the sense of travel times. Further, because we only consider sound-speed perturbations here, the primary impact on waveforms is to shift their phases and to a lesser degree, amplitude. In principle, we may also include amplitudes, instantaneous phase, or even raw waveform differences (e.g., Dahlen \& Baig 2002; Bozdağ et al. 2011; Rickers et al. 2013).

The basic goal in seismology is to relate properties of the interior to wavefield measurements at the bounding surface. The first step involves defining a misfit or cost functional that comprises some measure of the difference between measurement and prediction. An example of a misfit function $(\chi)$ in the case of time-distance helioseismology is the $L_{2}$ norm of the difference between measurement $\left(\tau^{\mathrm{o}}\right)$ and prediction $(\tau)$ at some set of locations $i$ (Hanasoge et al. 2011)

$$
\chi=\frac{1}{2} \sum_{i}\left(\tau_{i}-\tau_{i}^{\mathrm{o}}\right)^{2} .
$$

A more general formulation to include a noise-covariance matrix in the definition of the misfit is discussed by Hanasoge et al. (2011). Here, we study a simpler problem where the 
data are known exactly. The next step is to determine how to change the model so that the predicted travel times $\tau_{i}$ are closer to the measurements $\tau^{\mathrm{o}}$ in the sense of norm (1). This is a high-dimensional inverse problem, since we seek to alter various properties such as flows, sound speed and density of the 3-D interior, thereby introducing a large number of parameters, in order to appropriately alter the travel times measured at the bounding surface of the Sun.

The misfit function (11) depends on the model, i.e., $\chi=\chi(\mathbf{m})$, where $\mathbf{m}=\mathbf{m}(\mathbf{x})$ is the model of the Sun and $\mathbf{x}$ is the spatial coordinate. To vary the misfit, we consider the Taylor expansion of equation (11) around model $\mathbf{m}$,

$$
\delta \chi=\sum_{i}\left(\tau_{i}-\tau_{i}^{\mathrm{o}}\right) \frac{\partial \tau_{i}}{\partial \mathbf{m}} \delta \mathbf{m},
$$

and it is seen that to reduce the misfit, i.e., to induce $\delta \chi<0$, we first need access to the gradient of the misfit function $\partial \tau_{i} / \partial \mathbf{m}$. Gradient-based optimization methods are designed to address this question, specifically to minimize penalty (1), an inherently nonlinear function of the 3-D model parameters. The gradient of misfit (1) with respect to model parameters is the so-called 'sensitivity kernel', alternately known as the Fréchet derivative,

$$
\frac{\partial \tau_{i}}{\partial \mathbf{m}}=\mathbf{K}\left(\mathbf{x}, \mathbf{x}_{i} ; \mathbf{m}\right)
$$

where $\mathbf{K}$ is the sensitivity of travel time $\tau_{i}$ to changes in the model $\mathbf{m}=\mathbf{m}(\mathbf{x})$, and is therefore a function of the model and space. Equation (3) along with (2) gives us a prescription to compute a model that minimizes the misfit for the quiet Sun,

$$
\delta \chi=\int_{\odot} d \mathbf{x} K_{c} \delta \ln c+K_{\rho} \delta \ln \rho+\mathbf{K}_{\boldsymbol{v}} \cdot \delta \boldsymbol{v},
$$

where $c$ is sound speed, $\rho$ is density and $\boldsymbol{v}$ are flows, $K_{c}, K_{\rho}$, and $\mathbf{K}_{\boldsymbol{v}}$ are kernels for sound speed, density and flows respectively (Hanasoge et al. 2011, 2012). We use log quantities for variations in $c$ and $\rho$ since they are positive definite.

This article aims to introduce the basic concepts of this inverse methodology and is not exhaustive in its scope. We therefore limit ourselves to the study of a sound-speed inversion, described thus

$$
\delta \chi=\int_{\odot} d \mathbf{x} K_{c} \delta \ln c .
$$

To compute the misfit gradient $K_{c}$, we apply the adjoint method described by Hanasoge et al. (2011), used to simultaneously construct kernels $K_{c}, K_{\rho}$, and $\mathbf{K}_{\boldsymbol{v}}$ (also see e.g., Tarantola 1984; Tromp et al. 2005). However, we only retain $K_{c}$ for this problem. 
Seismic inversions are matrix-inverse problems of the form

$$
A \delta \mathbf{m}=\{\delta \tau\}
$$

where $A=A(\mathbf{m})$ is a fat matrix of dimension $N \times M$, and where the $M$ unknown model parameters are substantially larger than the $N$ measurements, $\delta \mathbf{m}$ is the model update vector, of size $M \times 1$ and $\{\delta \tau\}$ is an $N \times 1$ vector composed of the travel times. The matrix $A$ comprises the sensitivity of the travel time to model parameters, i.e., it is composed of sensitivity kernels. At present, inverse problems in local helioseismology focus on constructing sensitivity kernels using only 1-D vertical stratification, leading to lateral (horizontal) translation invariance. Although likely erroneous for certain problems, this approach is generally invoked regardless because a viable methodology to fully account for the three-dimensionality and non-linearity of the inverse problem has only recently been introduced (Hanasoge et al. 2011). Inverse approaches that rely on translation invariance possess the additional feature that the computational cost scales very weakly with the number of measurement points, unlike in the adjoint method. On the other hand, it is possible to mitigate the computational cost of adjoint-method based approaches by choosing a set of observation points such that coverage and resolution are maximized.

Matrix $A$ can be very big (with $10^{12}$ elements or more), and will possess a high condition number, and therefore inverting it is not an option. Consequently, we use an iterative procedure to arrive at some appropriate inverse of $A$ and therefore, $\delta \mathbf{m}$. To perform iterations, a local linear approximation is invoked, much as in the style of the Taylor expansion in equation (2), and methods such as steepest descent, conjugate gradient or the quasi-Newton limited-memory BFGS are applied.

The adjoint method, a means of obtaining gradients of the misfit function $\chi$, is well studied in the regime of relatively strong heterogeneities, as demonstrated by the successful application to terrestrial seismic inversions of, e.g., the Southern-California crust (Tape et al. 2009), European structure (Zhu et al. 2013) and Australia (Fichtner et al. 2009). This technique is applied to constrained optimization problems in which we seek to minimize the misfit with the constraint that the wavefield satisfy the partial differential equation that governs wave propagation in the Sun. We define the helioseismic operator,

$$
\rho \partial_{t}^{2} \boldsymbol{\xi}=\nabla\left(\rho c^{2} \boldsymbol{\nabla} \cdot \boldsymbol{\xi}+\rho g \xi_{z}\right)+\mathbf{g} \boldsymbol{\nabla} \cdot(\rho \boldsymbol{\xi})+\mathbf{S},
$$

where density is denoted by $\rho=\rho(\mathbf{x})$, sound speed by $c=c(\mathbf{x})$, gravity by $\mathbf{g}=-g(z) \hat{\mathbf{z}}$, the vector acoustic wave displacement by $\boldsymbol{\xi}=\boldsymbol{\xi}(\mathbf{x}, t)$, whose vertical component is $\xi_{z}$, the source by $\mathbf{S}=\mathbf{S}(\mathbf{x}, t)$ and time by $t$. The covariant spatial derivative is denoted by $\boldsymbol{\nabla}$ and the partial derivative with respect to time is $\partial_{t}$. The adjoint method relies 
on making predictions and using the difference with observations to drive changes in the solar model. Thus, we require a technique to solve equation (77). The pseudo-spectral solver SPARC developed by Hanasoge \& Duvall (2007); Hanasoge et al. (2008), fulfills the purpose of solving equation (7) in Cartesian geometry. Lateral (horizontal) derivatives are computed using Fourier transforms and the radial (vertical) derivative using a sixth-order accurate compact-finite-difference scheme (Lele 1992). Time stepping is achieved through the repeated application of an optimized second-order five-stage Runge-Kutta technique (Hu et al. 1996). We line the side and vertical boundaries with perfectly matched layers (Hanasoge et al. 2010) that effect high fidelity wave absorption.

The adjoint method consists of computing forward and adjoint wavefields. The forward calculation is a predictor step, making a prediction on the photospheric cross correlation (or some other measurement) along with the attendant 3-D seismic wavefield in the interior. This calculation captures the connection between the interior sensitivity of the wavefield and the surface seismic signature. The adjoint calculation consists of performing a 3-D wavefield simulation driven by the difference between prediction and observation, as measured by equation (11). Roughly speaking, this captures the connection between the interior and the measurement misfit as recorded at the surface. Finally, the time-domain convolution of forward and adjoint wavefields gives the total misfit gradient, i.e., all the desired sensitivity kernels (Eq. 4). Because this formulation of the adjoint method is numerical, forward and adjoint simulations may be carried out for arbitrary backgrounds. Further, with a few calculations, all relevant kernels may be simultaneously obtained. The analysis, kernel expressions and algorithm are outlined in sections 4, 5 and 6 respectively of Hanasoge et al. (2011). Finally, we note that the extension to a variety of other measurements such as resonant frequencies closely follows the analysis in section 4 of Hanasoge et al. (2011), with the relevant measurement framed in a manner so as to connect it to Green's functions of the medium.

Waves in the Sun are excited in a thin near-surface radial envelope (e.g., Stein \& Nordlund 2000) but uniformly in the lateral (horizontal) direction. Thus the helioseismic wavefield is excited by distributed sources, which, together with the stochastic nature of the excitation, makes the calculation of sensitivity kernels complicated (Hanasoge et al. 2011). This is because the wavefield measured at a given point consists of contributions from a wide range of sources and the cross-correlation of the wavefield measured at a point pair thus averages these contributions. However, in the case where the distribution of sources is uniform, the cross-correlation can be shown to be closely related to Green's function of the medium (e.g., Snieder 2004). This correspondence allows for treating the second-order cross-correlation measured between a point pair as arising from a deterministic, single 
source-receiver configuration, greatly reducing the complexity of the problem (the point pair map on to the source and receiver). While it may appear that the solar wavefield is an ideal fit for this correspondence (owing to the lateral uniformity of sources), the damping mechanism and the line-of-sight nature of observations diminish the accuracy of the relationship (e.g., Gizon et al. 2010). However, it still serves as a very useful first approximation to study the simplified deterministic source-receiver problem since it allows for the appreciation and development of inverse methodology prior to comprehensive modeling. Kernels in this limit treat each branch of the cross correlation measured between a pair of points as the wave displacement due to a deterministic single source.

\section{The inversion}

The road to obtaining consistent inversions is long, requiring a number of important steps to be implemented. Here we discuss practical issues and the choices we have made. We do not start from a vacuum, and indeed, there exists significant geophysical seismic literature on these topics, and the choices from these articles guide our thinking. However, the helioseismic inverse problem possesses its own idiosyncrasies and to optimize our methodology, an exhaustive survey of these choices will be necessary. This is especially the case when including more parameters such as flows and magnetic fields.

\subsection{True and starting models}

The goal is to invert for the true anomaly in sound speed shown in Figure1. Also shown in Figure 1 is the starting model, which is a solely vertically stratified, convectively stabi-

lized form of model S (Christensen-Dalsgaard et al. 1996; Hanasoge 2007; Hanasoge et al. 2008). Sound-speed perturbations shown in Figure are measured as deviations from this 'quiet Sun' stratification, i.e., $\left[c(x, z)-c_{q}(z)\right] / c_{q}(z)$, where $c_{q}$ is the nominal sound-speed in the quiet Sun and $c(x, z)$ is the sound speed of the current model. To accelerate convergence, we may also constrain the surface layers in the starting model to be identical to those of the true model, the argument being that the surface layers of the true model would be 'observable' (which we do in Section 2.10). For now, we choose the starting model, $c(x, z)=c_{q}(z)$. In the subsequent discussion and in various Figures and attendant captions, we will make use of the following definition

$$
\delta \ln c=\ln \frac{c(x, z)}{c_{q}(z)} .
$$




\subsection{Master and slave pixels}

Recalling the discussion on source-receiver pairs in the preceding section, we term sources as master pixels and receivers as slaves. Tromp et al. (2010) and Hanasoge et al. (2011) showed that the cost of inversion scales with the number of master pixels and hence the nomenclature. Thus having selected points at which to place sources (master pixels), we may increase the number of receivers (slaves) arbitrarily without accruing additional computational cost. Choices for master pixels are therefore crucial since we would like to maximize seismic information. There are likely more formal and rigorous ways to make this choice but in the effort here, we have discovered through the process of trial and error that placing master pixels in the near field of the perturbation leads to faster convergence. We thus choose 7 master pixels placed at points along the sound-speed perturbation as shown in Figure 1. In order to introduce more seismic information, we perform a few iterations for a given set of master pixels and replace these by another set. In the inversion presented here, the master pixels change from the originally chosen set (indicated by triangles in Figure 10 to another set of 7 pixels at iteration 7, indicated by asterisks. The new set of pixels is more sparsely distributed and is spread out over a larger horizontal distance, to improve the imaging aperture. We do not introduce further changes to the set of masters because seismic information is concentrated in the vicinity of the perturbation, which we explore thoroughly with the overall set of pixels. Slave pixels may also be changed from iteration to iteration, but here, we have maintained the same set of receivers throughout the inversion.

\subsection{Measurements}

We measure wave travel times between point pairs. Using the definition of the linear travel time as set out by Gizon \& Birch (2002), we formulate the adjoint method for this measurement (Hanasoge et al. 2011). In practice, the relative travel time between two waveforms is measured by actually cross correlating them and extracting the time lag associated with the peak correlation coefficient. For instance, if waves appear at point B at a positive time lag in relation to point $A$, then point $B$ acts as the receiver (slave) to source A (master). In Figure 2, we show the time-distance diagram for a source at $x=-15$ $\mathrm{Mm}$. We measure travel times for $p$ modes over a range of point-pair distances for the first, second and third bounces over specified frequency bands. 

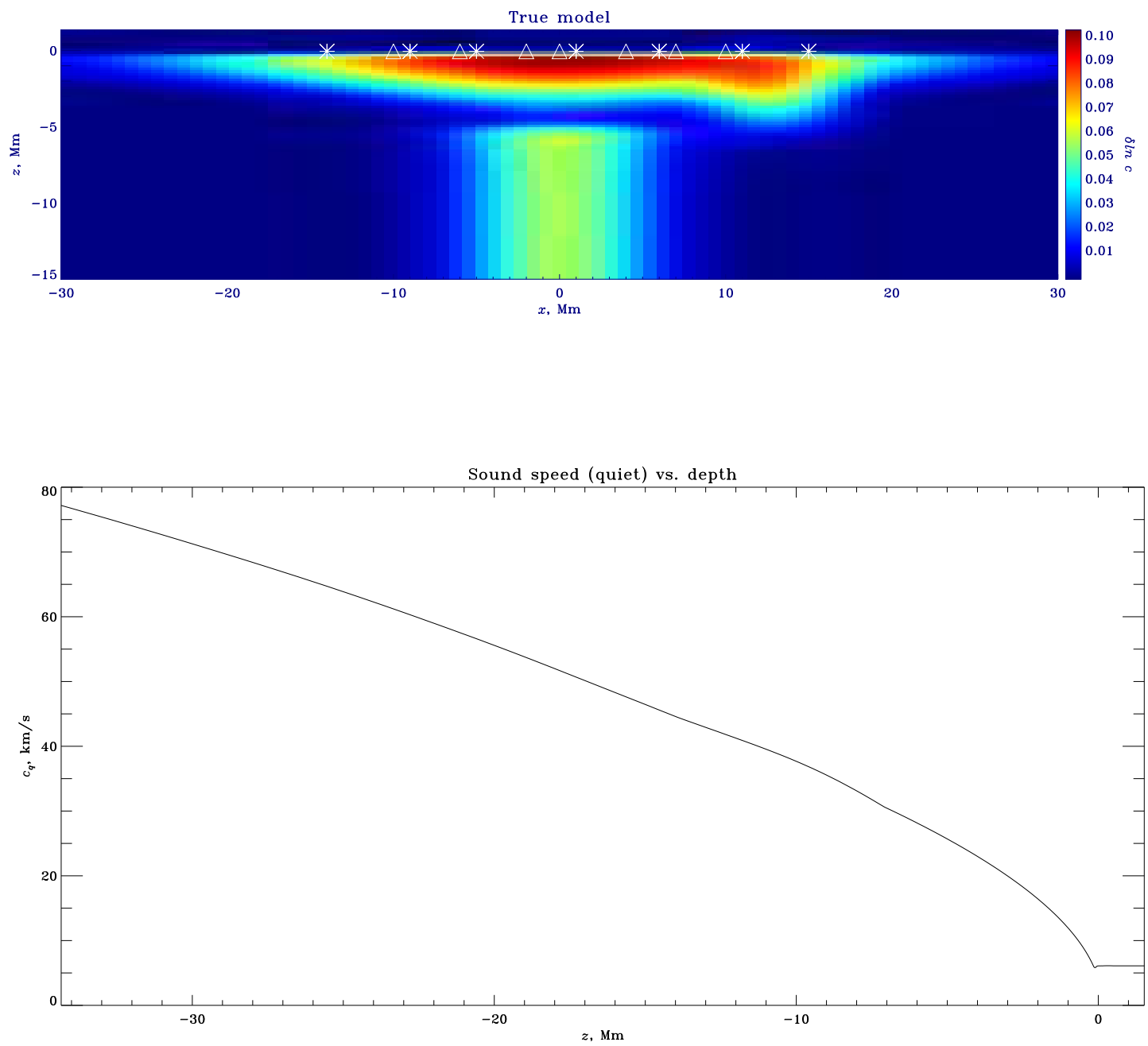

Fig. 1.- True model (upper panel), where $\delta \ln c$ is defined in equation (8), and the quietSun sound-speed, $c_{q}(z)$ in the lower panel. The triangles denote the first set of master pixels (sources) and the asterisks the second set. The master pixels are switched at iteration 7 , to introduce new seismic information. Because wave excitation occurs in the very nearsurface layers of the Sun $(z=-50 \mathrm{~km})$, we fix the location in depth but are free to vary the horizontal location. 


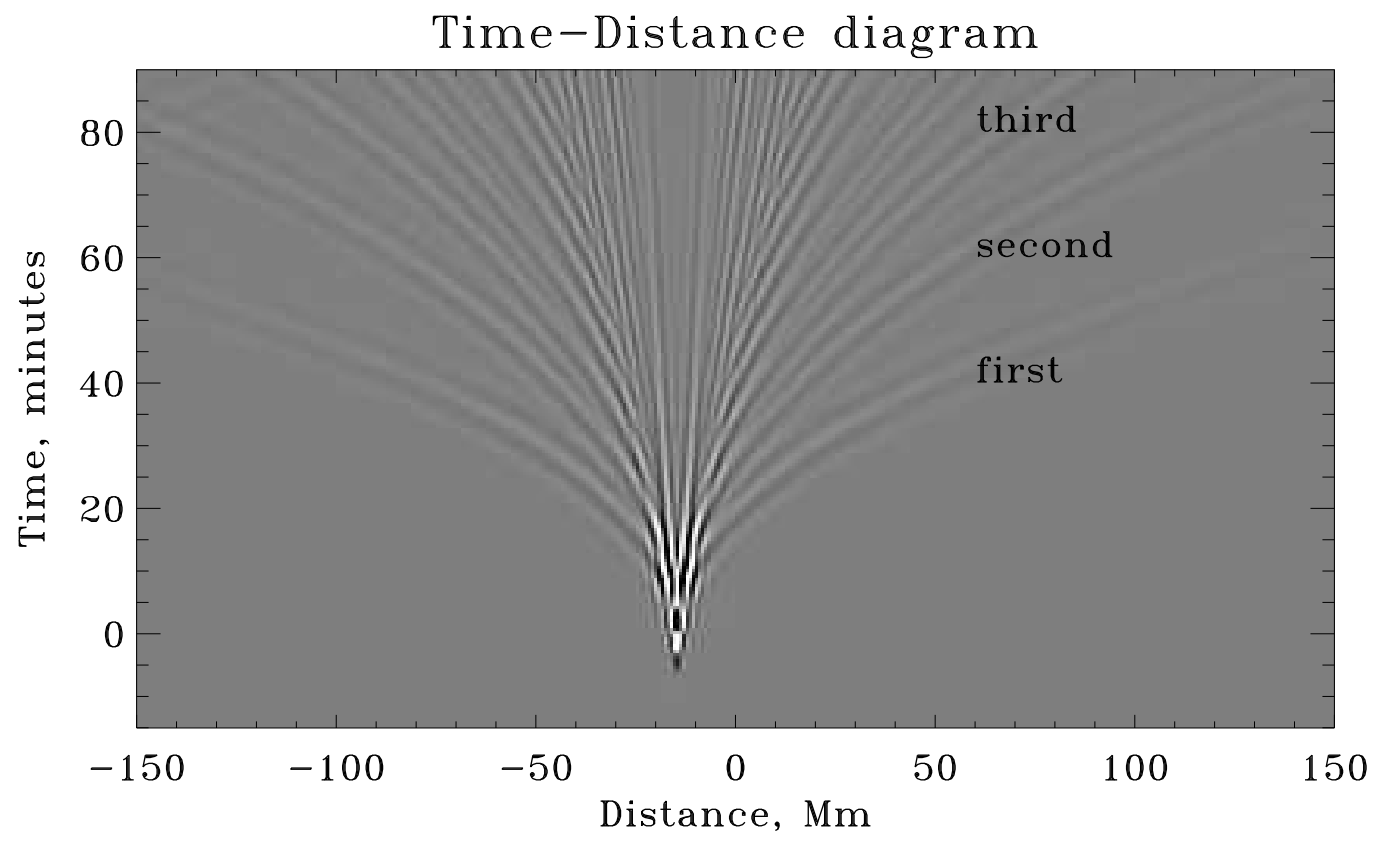

Fig. 2.- Time-distance diagram. The master pixel (source) in this case is placed at $x=-15 \mathrm{Mm}$. Travel time shifts measured at slave pixels (receivers) for a given bounce (first, second, or third) are used in the inversion. In order to distinguish between the various arrivals, we select receivers that are at a minimum distance of $15 \mathrm{Mm}$ away from the source for the first and second bounces and $30 \mathrm{Mm}$ for the third bounce.

\subsection{Adjoint source}

For a given source point, we measure travel times at receivers located farther than $15 \mathrm{Mm}$ from it. This minimum separation allows for the distinction between the various bounces of $p$ modes. At distances shorter than $15 \mathrm{Mm}$, it is no longer possible to clearly interpret the measurement. We only simulate for $1.5 \mathrm{hrs}$ of solar time, which places a restriction on a maximum source-receiver distance possible for each bounce. In the adjoint calculation, the wave equation is forced with adjoint sources placed at all the receiver locations where measurements are made. The adjoint source at any given measurement point consists of the travel-time shift multiplied by the time reverse of the temporal derivative of 
the measured waveform from the forward calculation. In Figure 3, the full adjoint source is shown in the upper panel and a cut at a fixed spatial location is shown in the bottom.
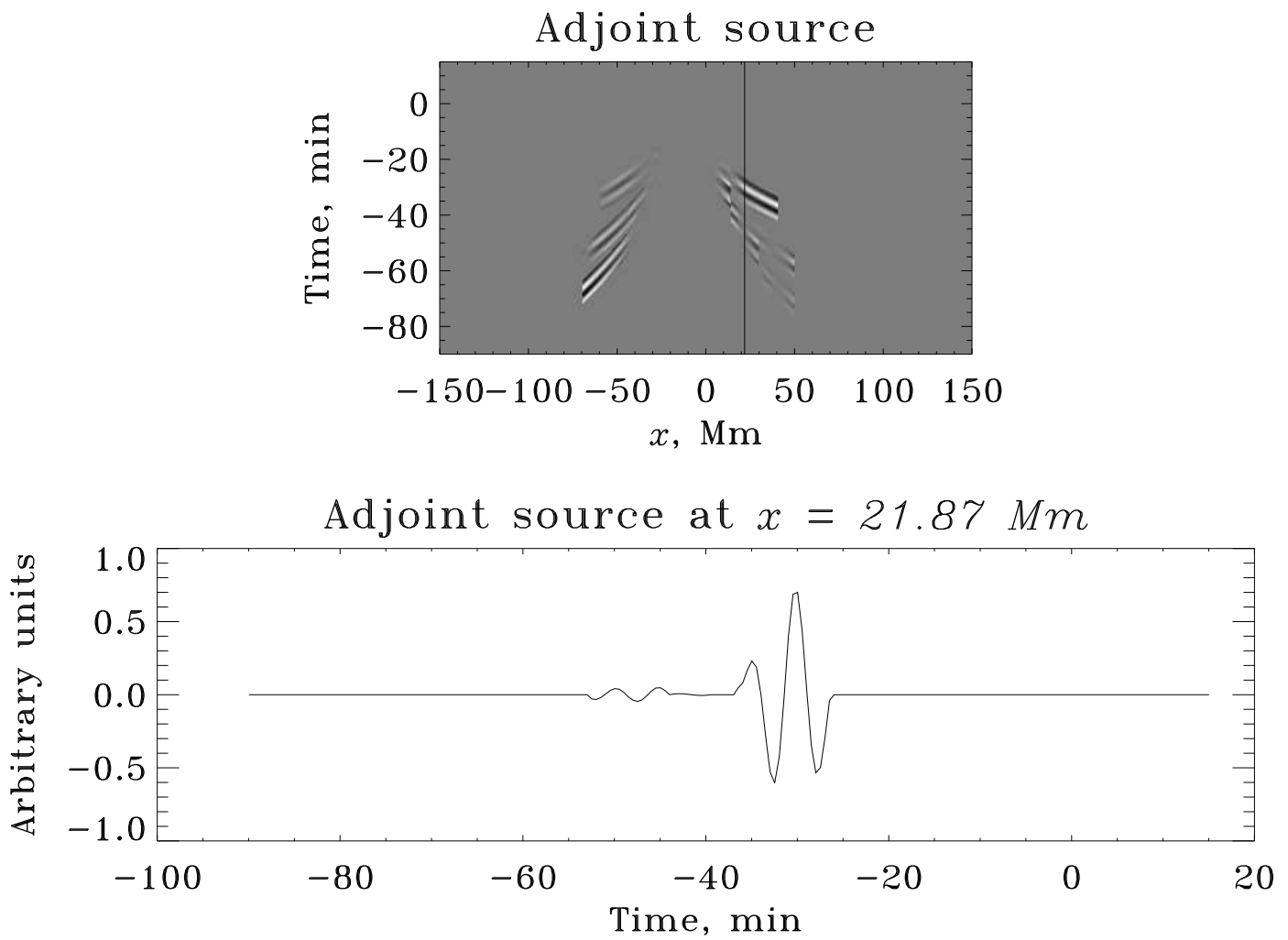

Fig. 3.- Adjoint sources at receivers (upper panel) corresponding to the master pixel shown in Figure 2, Each adjoint source is the time-reversed temporal derivative of the waveform measured at that receiver, multiplied by the cross-correlation travel time shift. The adjoint source at a specific $x$ location is shown in the lower panel. The waveform multiplied by the travel-time shift is the largest for the first bounce, which, owing to time reversal, appears at a later time in the adjoint source. The adjoint source suggests that the most significant travel-time deviations are recorded by the first bounce, thereby playing a prominent role when constructing the gradient. 


\subsection{Discrete adjoint method}

In the formulation adopted here, the adjoint method is treated in a continuous sense

(Hanasoge et al. 2011), and expressions for kernels that are computed by convolving the forward and adjoint wavefields are derived for continuous space. However, numerical simulations are performed on discrete grids, and indeed, errors are generated when the continuous adjoint formulation is discretized. The gradient thus obtained is not as accurate as when the problem is posed consistently in the discrete sense. This slows down convergence and is a well noted issue in these seismic inverse problems (for airfoil design, see e.g., Giles \& Pierce 2000). Nevertheless, because convergence is observed and because there is no easy or obvious route to a discrete adjoint formulation, we proceed with the (inaccurate) continuous analog.

\subsection{Preconditioning and Smoothing}

While adjoint methods may not explicitly state the role of regularization, it does make its way into the heart of the problem. At every iteration, the total misfit gradient, summed over all master pixels, contains non-smooth variations co-spatial with source locations, which may slow convergence. To mitigate this problem, spatial smoothing must be applied to the gradient.

The rate of convergence can be improved by 'preconditioning' the gradient, which in practice involves multiplying the gradient by a suitable function termed the preconditioner, i.e., the gradient is preconditioned first and spatially smoothed next. The sensitivity of the convergence rate to different types of preconditioners was studied by Luo et al. (2013), who found that the optimal preconditioner for the problem they were studying was a convolution of the time derivatives of the forward and adjoint wave fields (see their Eqs. [108] and [109]). However, we found that preconditioning (based on the methods of Luo et al. 2013) and smoothing led to slower convergence rate in comparison to just smoothing. The design and application of preconditioners to helioseismology is deferred to the future and we restrict ourselves only to smoothing the gradient here. Note that explicit regularization terms (user prescribed) may indeed be included in the original statement of the problem, since the adjoint method is designed to address constrained-optimization problems. 

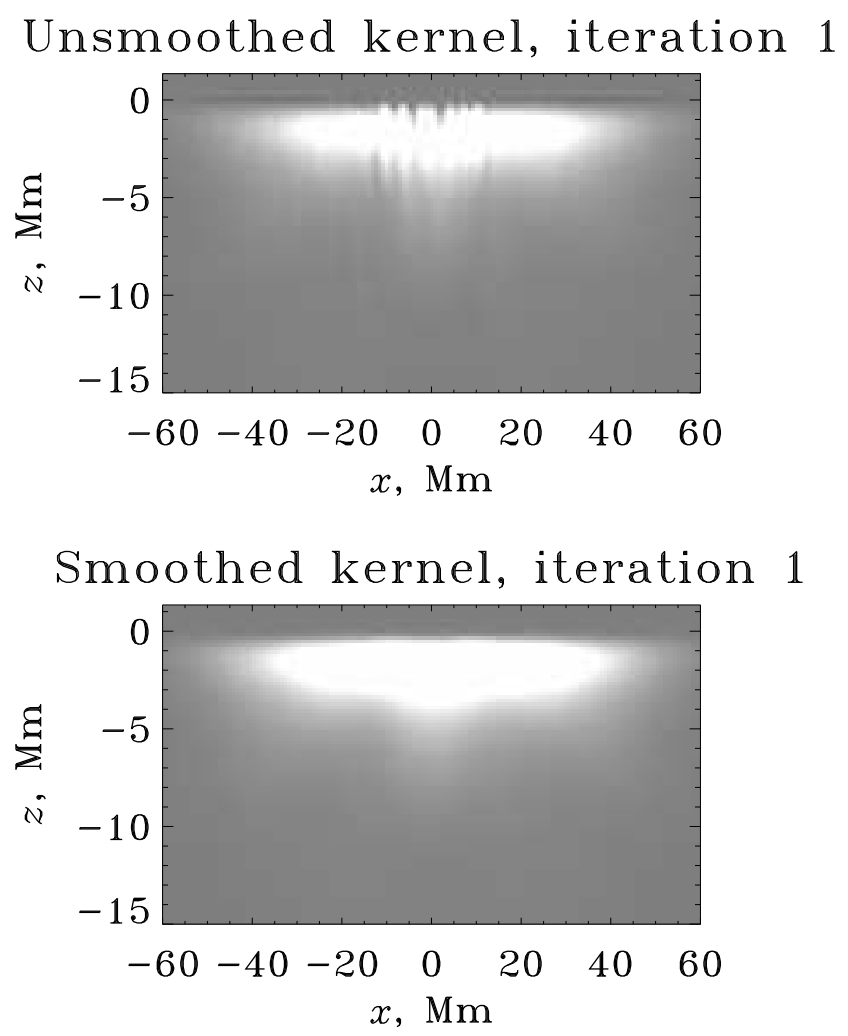

Fig. 4.- The raw sound-speed gradient, shown in the upper panel has sharp variations due to numerical issues related to the spatially localized forward source. The smoothed kernel is shown in the lower panel, where a 3-point Gaussian filter was applied to accomplish smoothing. The update is then computed through $c_{02}=c_{01}\left(1+\varepsilon \overline{K_{c_{01}}}\right)$, where the overbar indicates smoothing, $c_{02}$ is the sound-speed model for the second iteration and $\varepsilon$ is a small constant.

\section{7. $\quad$ Model updates}

Given the gradient, the model can be updated using a variety of methods. The first iteration relies on steepest descent, in which the update is tangent to the gradient direction. At higher iterations, we may choose between conjugate gradient and L-BFGS to create updates. Conjugate gradient requires the previous and current gradients to form the update where L-BFGS can be designed to use the full history of gradients and models to create an update. Although not shown here, from preliminary testing we find that L-BFGS and conjugate gradient converge at roughly the same rate. More careful testing may reveal the 
parameter regimes where one method is faster than the other.

Since we only consider sound-speed perturbations, the smoothed sound speed sensitivity kernel is first normalized by its largest absolute value so that it $\left(\bar{K}_{c_{i}}\right)$ spans the range $[-1,1]$. We then perform a line search, using 5 different models, $c_{i+1}=c_{i}\left(1+\varepsilon \bar{K}_{c_{i}}\right)$, where $c_{i}$ is the model at the $i$ th iteration, $\varepsilon$ is a small constant that takes on values $[0.01,0.02,0.03,0.04,0.05]$. Every value of $\varepsilon$ leads to a model $c_{i+1}$, and we estimate the misfit for each. At every iteration, we test for local convexity by performing a line search. Typically an elegant $L$-curve is observed, as in Figure 5. We choose the model corresponding to the minimum point of this curve as the model for the next iteration, i.e. the update corresponds to the valley of the line search curve. The update parameter $\varepsilon$ generally decreases with iteration, and $\varepsilon$ for updates to successive models is smaller in magnitude. Typically, $\varepsilon \sim 0.06$ for the very first iteration and then drops to about $\varepsilon \sim 0.004$ at the eleventh iteration.

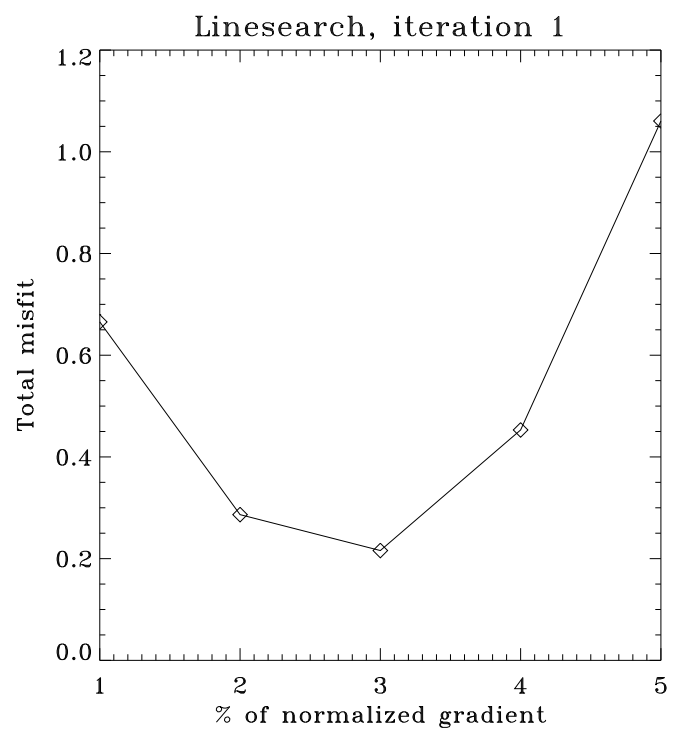

Fig. 5. - Line search at each iteration to determine $\varepsilon$ for the update $c_{i+1}=c_{i}\left(1+\varepsilon \bar{K}_{c_{i}}\right)$. The $x$ axis shows different values of $\varepsilon$ and the $y$ axis the misfit associated which the corresponding model. In this case, we choose the model for which the misfit reaches a minimum, i.e., for $\varepsilon=0.03$.

Every few iterations, the $L$-curve for a non-steepest-descent method is not easily produced. In such scenarios, we revert to steepest descent as a means of 'resetting' the inversion. For instance, we might have the following configuration of updates - 1 - steepest, 2 , 
3, 4 - conj. grad., 5 - steepest, 6, 7 - conj. grad, where the numbers indicate the iteration index. We show 12 iterations of an inversion for the setup discussed in Figure 1 using a combination the conjugate gradient and steepest descent methods in Figure 6. We also applied the L-BFGS algorithm after 4 iterations of steepest descent but found the rate of convergence to be generally unchanged. The performance of the method appears to be less sensitive to these choices and much more to the introduction of external information (such as surface constraints, new pixels etc.).

\subsection{Uniqueness}

In high-dimensional inverse problems, the choice of the starting model and type of measurements introduced to update the model may be critical to avoiding being trapped in a local minimum. A standard strategy applied to mitigate the chances of encountering this undesirable outcome is to first use measurements taken from low frequency modes and gradually introduce higher frequencies as the model iteratively accrues features. This particular issue can be very serious when attempting to image reflectors in the interior, as in exploration geophysics, but it is unlikely to be critical for helioseismology. Because the frequency range of trapped modes in the Sun is so narrow $(2.5-5.5 \mathrm{mHz})$, we choose here to utilize the entire passband. Indeed, we are aware that this strategy may not be optimum for all applications but we find it to be successful in the case of sound-speed perturbations studied here.

\subsection{Testing convergence}

To verify that misfit is being minimized for all the measurements, we measure the misfit associated with each model for travel times binned into categories by their bounce number (first, second or third) and frequency band $(2.5-4,2.5-5,2.5-5.5)$. Note that we could also have measured the misfit using ridge- and phase-filtering to isolate modes in various parts of the power spectrum but our categories are simpler in this case. Thus we confirm that the misfit is uniformly reduced in these 9 categories. A similar strategy has been used successfully in terrestrial applications, e.g., Zhu et al. (2013) although because terrestrial seismic waves exhibit a larger temporal frequency range, they apply frequency filters to their data. Fixing the lower frequency cutoff, Zhu et al. (2013) increase the upper corner of the bandpass with iteration, gradually allowing in more information as the model grows in complexity. We also calculate the model misfit by computing the $L_{2}$ norm of the 
difference between the true and inverted models as a function of iteration. Both data and model misfit are seen to decrease with iteration in Figure 7.

\subsection{Including "surface" constraints}

The sound-speed anomaly studied here has a 'surface' signature and we can include this as a constraint on the model. It is of relevance because in reality, perturbations such as supergranules, meridional circulation, sunspots and active regions are optically observed

at the photosphere and these observations can be used to accelerate convergence. For the inverse problem at hand, $p$ modes are used to image the sound-speed perturbation. Surface-gravity $f$ modes, which are very sensitive to the surface, do not register soundspeed perturbations since the restoring force for these waves is gravity and not pressure. Consequently, adding a surface constraint to the inversion is likely to accelerate convergence for this inverse problem.

In Figure 8, we see direct evidence of this, where the bottom-left panel shows a smooth decline in model misfit with iteration, unlike in Figure 6, which displays a non-monotonic trajectory. Overall, both data and model misfit are lower in Figure 8 in comparison to Figure 6. We also over plot all the misfit categories in Figure 9 to highlight the (anticipated) superiority of surface-constrained inversions.

Finally, we show the improvement between waveforms derived from "data" and the model in Figure 10, By iteration 11, the waveforms start matching up well.

\section{Discussion}

Full waveform inversion provides a means of addressing longstanding problems in helioseismology. It directly addresses the major issue of non-linear dependencies of travel times on properties of the solar medium in structures such as sunspots and supergranules. While iterative inversions are indeed possible using ray theory as the forward model, wave propagation is demonstrably not well captured in this high-frequency approximation (Birch et al. 2001). Helioseismology is increasingly a high-precision science and to make accurate inferences, it is important to model wave effects as fully as possible. Finite frequency forward calculations of the helioseismic wavefield are now routinely performed, and in this article we have discussed full waveform inversion strategies within this context.

A basic lacuna of current approaches to $3-\mathrm{D}$ helioseismic inversions is that there is 
rarely a consistency check of how much the inverted model reduces the misfit between seismic prediction and observation. At each iteration in our inversion, we perform a line search to determine how much to change model, and generally find that beyond $3-5 \%$ the misfit actually rises, suggesting that the linear connection between misfit and model change is restricted to this regime. Of course, the caveat in drawing this conclusion is that our inversion method is either quasi-Newton- or conjugate gradient based, whereas prior helioseismic inversions have relied on Gauss-Newton-based approaches. In general, Gauss-Newton allows for taking larger steps in model space but it must be emphasized again that the actual extent to which misfit is reduced has generally not been measured. The closest to a consistent inversion can be attributed to Cameron et al. (2008), who attempted to study a set of sunspot models using linear magneto-hydrodynamic numerical simulations to determine how well observations can be matched. In a purely forward approach ("probabilistic"), the model space is exhaustively searched, determining the misfit for each model. However, given the computational expense for full wave modeling codes, this may be an infeasible approach.

The methodology discussed here still requires development and a more careful exploration of techniques that can enhance convergence. Purely computational test problems, such as the inversion for flows and magnetic fields, will be the focus of future studies. However, full waveform inversion provides a firm theoretical foothold for a field that has long sought a means to accurately interpret helioseismic measurements. The hope is that, with the simultaneous development of inverse theory and high-fidelity numerical methods to rapidly simulate wave propagation in a medium that closely mimics the Sun, we may finally able to settle issues of great relevance to understanding solar dynamics.

S.M.H. acknowledges funding from NASA grant NNX11AB63G. We also thank Hejun Zhu for his useful insights on FWI methodology.

\section{A. Adjoint source}

We use equation (4) from Gizon \& Birch (2004) in order to define the weight function $W_{i}(t)$ for the travel-time measurement

$$
W_{i}(t)=-\dot{\mathcal{C}}_{i}^{\mathrm{p}}(t) \frac{f(t)}{\Delta t \sum_{t^{\prime}} f\left(t^{\prime}\right)\left[\dot{\mathcal{C}}_{i}^{\mathrm{p}}\left(t^{\prime}\right)\right]^{2}},
$$


where $\mathcal{C}^{\mathrm{p}}$ is the predicted waveform (cross correlation), $\Delta t$ is the temporal rate at which the waveform is sampled, $f(t)$ is a window, and the travel-time shift $\Delta \tau$ is given by

$$
\Delta \tau_{i}=\int d t W_{i}(t)\left(\mathcal{C}_{i}^{\mathrm{p}}-\mathcal{C}_{i}^{\mathrm{o}}\right)
$$

The adjoint source is given by

$$
f^{\dagger}(\mathbf{x}, t)=\sum_{i} \Delta \tau_{i} W_{i}(-t) \delta\left(\mathbf{x}-\mathbf{x}_{i}\right)
$$

where $\mathbf{x}_{i}$ is the a receiver (slave) and the summation is over all receivers.

\section{B. Steepest descent, Conjugate gradient and L-BFGS}

In all the methods described here, the model is updated thus, $\mathbf{m}^{k+1}=\mathbf{m}^{k}+\varepsilon \mathbf{p}^{k}$, where $\varepsilon$ is obtained through a line search, i.e., $\varepsilon$ that minimizes $\chi\left(\mathbf{m}^{k}+\varepsilon \mathbf{p}^{k}\right)$. Given the smoothed gradient at iteration $k, \mathbf{g}^{k}$. The steepest descent update is simply $\mathbf{p}^{k}=-\mathbf{g}^{k}$. The conjugate gradient update is given by

$$
\mathbf{p}^{k}=-\mathbf{g}^{k}+\beta^{k} \mathbf{p}^{k-1}, \quad \beta^{k}=\frac{\mathbf{g}^{k} \cdot\left(\mathbf{g}^{k}-\mathbf{g}^{k-1}\right)}{\mathbf{g}^{k} \cdot \mathbf{g}^{k}},
$$

and because there is a dependence on $\mathbf{p}^{k-1}$, the first iteration cannot also be performed by conjugate gradient.

The limited-memory BFGS update at iteration $N$ is obtained by manipulating the prior $m$ gradients and models. The limited-memory aspect of this is accomplished by sweeping forward and reverse through prior gradients.

$$
\begin{aligned}
& k=N \quad \mathbf{h}=\mathbf{g}^{k} \\
& \text { For } \mathrm{k}=\mathrm{N}-1, \mathrm{~N}-2, \ldots ., \mathrm{N}-\mathrm{m} \\
& \alpha^{k}=\frac{\left(\mathbf{m}^{k}-\mathbf{m}^{k-1}\right) \cdot \mathbf{h}}{\left(\mathbf{m}^{k}-\mathbf{m}^{k-1}\right) \cdot\left(\mathbf{g}^{k}-\mathbf{g}^{k-1}\right)} \\
& \mathbf{h}=\mathbf{h}-\alpha^{k}\left(\mathbf{g}^{k}-\mathbf{g}^{k-1}\right),
\end{aligned}
$$

$$
\begin{aligned}
& \text { For } \mathrm{k}=\mathrm{N}-\mathrm{m}, \mathrm{N}-\mathrm{m}+1, \ldots, \mathrm{N}-1 \\
& \alpha^{k}=\alpha^{k}-\frac{\left(\mathbf{g}^{k}-\mathbf{g}^{k-1}\right) \cdot \mathbf{h}}{\left(\mathbf{m}^{k}-\mathbf{m}^{k-1}\right) \cdot\left(\mathbf{g}^{k}-\mathbf{g}^{k-1}\right)} \\
& \mathbf{h}=\mathbf{h}+\alpha^{k}\left(\mathbf{m}^{k}-\mathbf{m}^{k-1}\right)
\end{aligned}
$$


The update is given by $\mathbf{p}^{N}=-\mathbf{h}$. The rule of thumb is to use between 3 and 7 prior gradients to construct the update, i.e., $3 \leq m \leq 7$ in equations (B2) (B3) $)$.

\section{REFERENCES}

Birch, A. C., Kosovichev, A. G., Price, G. H., \& Schlottmann, R. B. 2001, ApJ, 561, L229

Bozdağ, E., Trampert, J., \& Tromp, J. 2011, Geophysical Journal International, 185, 845

Cameron, R., Gizon, L., \& Duvall, Jr., T. L. 2008, Sol. Phys., 251, 291

Christensen-Dalsgaard, J., Dappen, W., Ajukov, S. V., Anderson, E. R., Antia, H. M., Basu, S., Baturin, V. A., Berthomieu, G., Chaboyer, B., Chitre, S. M., Cox, A. N., Demarque, P., Donatowicz, J., Dziembowski, W. A., Gabriel, M., Gough, D. O., Guenther, D. B., Guzik, J. A., Harvey, J. W., Hill, F., Houdek, G., Iglesias, C. A., Kosovichev, A. G., Leibacher, J. W., Morel, P., Proffitt, C. R., Provost, J., Reiter, J., Rhodes, Jr., E. J., Rogers, F. J., Roxburgh, I. W., Thompson, M. J., \& Ulrich, R. K. 1996, Science, 272, 1286

Dahlen, F. A., \& Baig, A. M. 2002, Geophysical Journal International, 150, 440

Dombroski, D. E., Birch, A. C., Braun, D. C., \& Hanasoge, S. M. 2013, Sol. Phys., 282, 361

Duvall, Jr., T. L., Jefferies, S. M., Harvey, J. W., \& Pomerantz, M. A. 1993, Nature, 362, 430

Fichtner, A., Kennett, B. L. N., Igel, H., \& Bunge, H.-P. 2009, Geophysical Journal International, 179, 1703

Giles, M. B., \& Pierce, N. A. 2000, Flow, Turbulence and Combustion, 65, 393

Gizon, L., \& Birch, A. C. 2002, ApJ, 571, 966

-. 2004, ApJ, 614, 472

—. 2005, Living Reviews in Solar Physics, 2, 6

Gizon, L., Birch, A. C., \& Spruit, H. C. 2010, ARA\&A, 48, 289

Hanasoge, S., Birch, A., Gizon, L., \& Tromp, J. 2012, Physical Review Letters, 109, 101101 
Hanasoge, S. M. 2007, PhD thesis, Stanford University, California, USA

Hanasoge, S. M., Birch, A., Gizon, L., \& Tromp, J. 2011, ApJ, 738, 100

Hanasoge, S. M., Couvidat, S., Rajaguru, S. P., \& Birch, A. C. 2008, MNRAS, 391, 1931

Hanasoge, S. M., \& Duvall, Jr., T. L. 2007, Astronomische Nachrichten, 328, 319

Hanasoge, S. M., Komatitsch, D., \& Gizon, L. 2010, A\&A, 522, A87

Hu, F. Q., Hussaini, M. Y., \& Manthey, J. L. 1996, Journal of Computational Physics, 124,177

Jackiewicz, J., Birch, A. C., Gizon, L., Hanasoge, S. M., Hohage, T., Ruffio, J.-B., \& Švanda, M. 2012, Sol. Phys., 276, 19

Lele, S. K. 1992, Journal of Computational Physics, 103, 16

Luo, Y., Modrak, R., \& Tromp, J. 2013, in Handbook of geomathematics, 2nd edn., ed. W. Freeden, M. Z. Nashed, \& T. Sonar (Springer Verlag)

Rickers, F., Fichtner, A., \& Trampert, J. 2013, Earth and Planetary Science Letters, 367, 39

Snieder, R. 2004, Phys. Rev. E, 69, 046610

Stein, R. F., \& Nordlund, Å. 2000, Sol. Phys., 192, 91

Tape, C., Liu, Q., Maggi, A., \& Tromp, J. 2009, Science, 325, 988

Tarantola, A. 1984, Geophysical Prospecting, 32, 998

Tromp, J., Luo, Y., Hanasoge, S., \& Peter, D. 2010, Geophysical Journal International, 183,791

Tromp, J., Tape, C., \& Liu, Q. 2005, Geophysical Journal International, 160, 195

Švanda, M., Gizon, L., Hanasoge, S. M., \& Ustyugov, S. D. 2011, A\&A, 530, A148

Zhu, H., Bozdağ, E., Duffy, T. S., \& Tromp, J. 2013, Earth and Planetary Science Letters, 381,1 

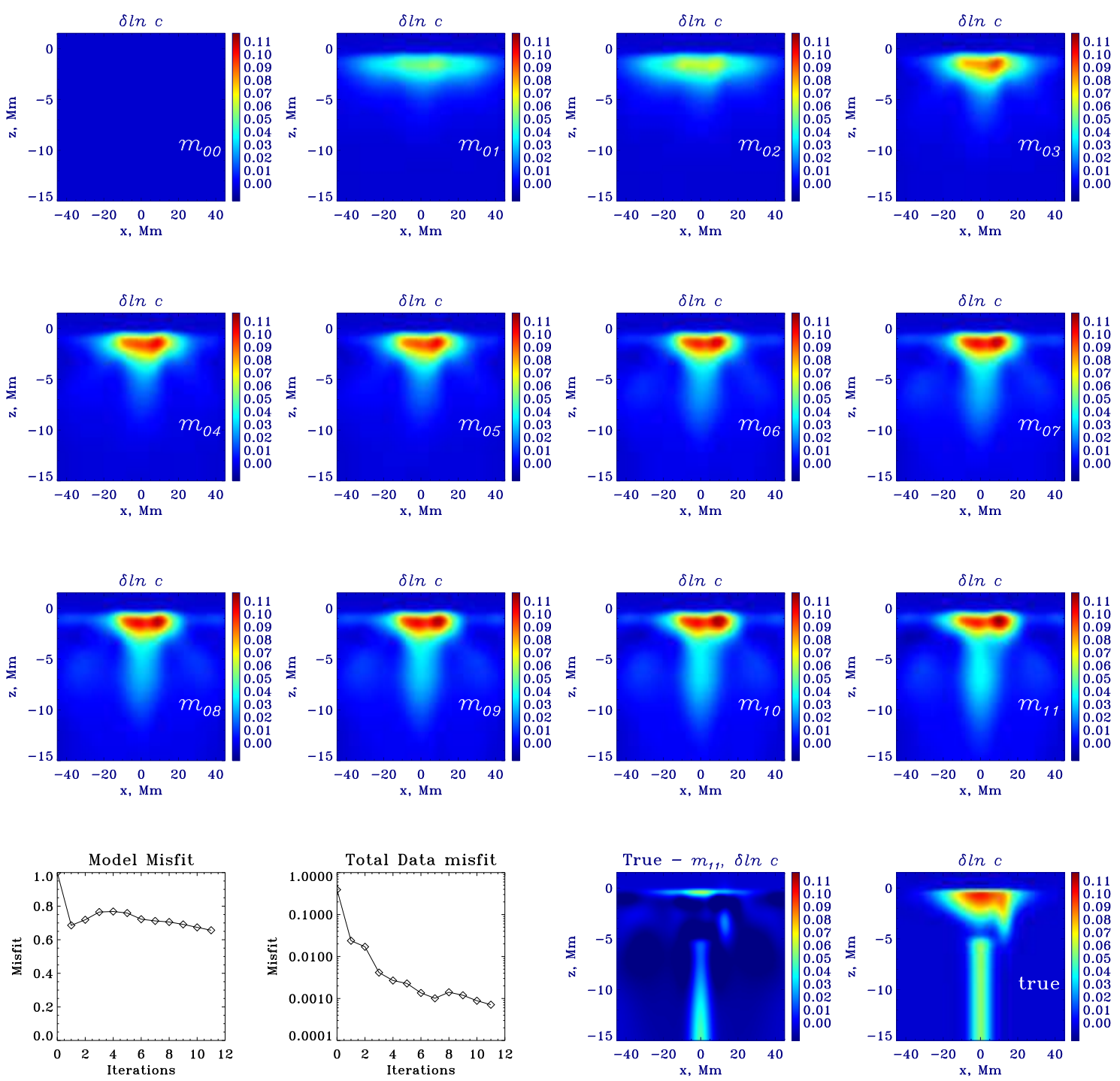

Fig. 6.- Iterations in a conjugate-gradient based inversion. The first iteration is performed using steepest descent and a combination of conjugate gradient and steepest descent are used to compute subsequent models. At iteration 7 , we change the set of master pixels and this creates a local jump in the data misfit because more information has been introduced. It is seen that models approach the true anomaly gradually but the reduction in both data and model misfits slows down with iteration. The model misfit is the normalized $L_{2}$ norm difference between the true and current model whereas the total data misfit is the same as equation (1). In the first few iterations, the model misfit increases because surface layers contain significant errors and $p$ modes possess limited sensitivity to these layers. As the model evolves it overcomes this local hill, appearing to 'fix' the surface layers, and a steady decline is seen in the last few iterations. 

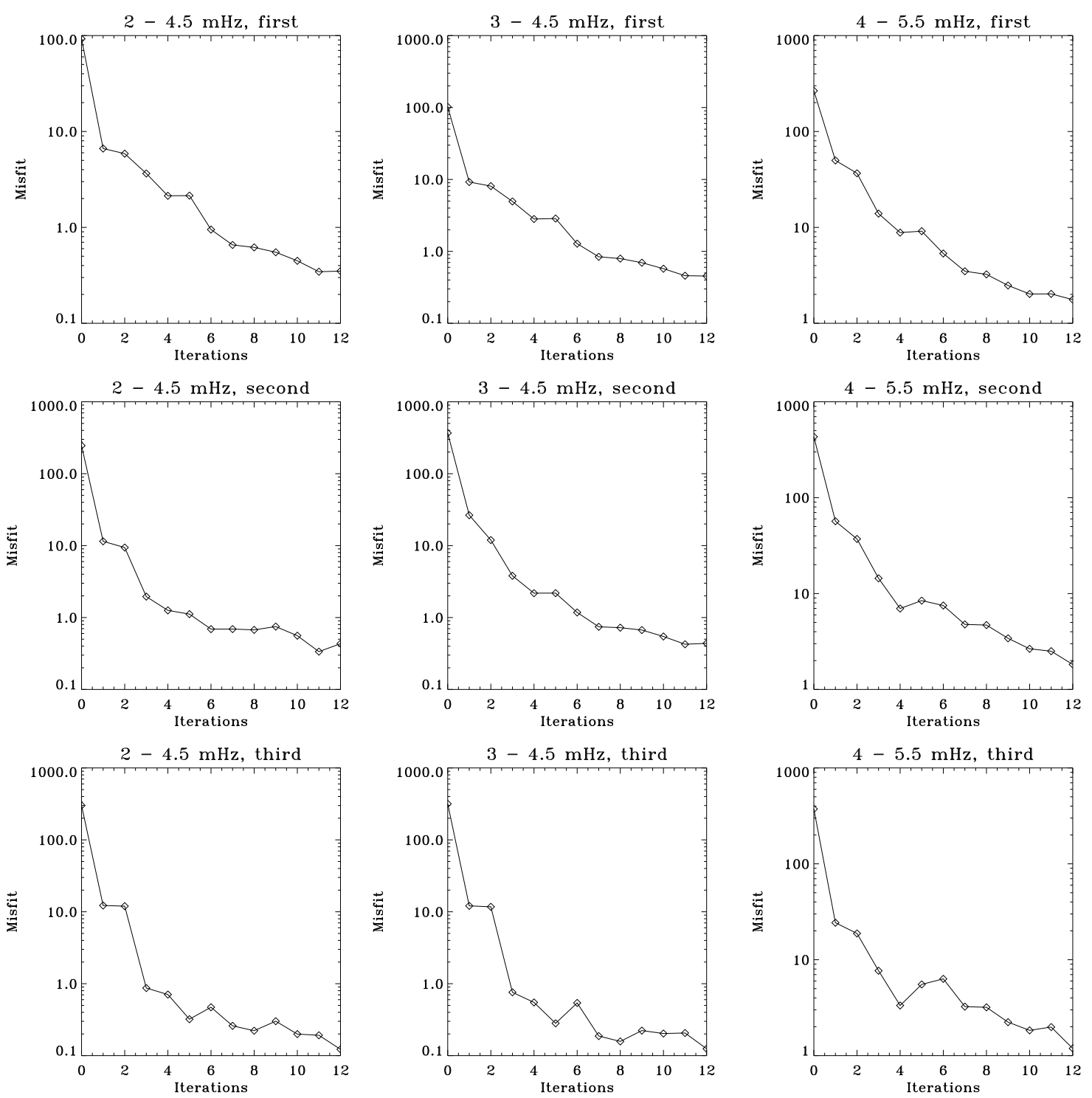

Fig. 7.- Misfit reduction with iteration, broken up based on the frequency bands and bounces. It is seen that regardless of the band, the misfit decreases uniformly (straying from monotonic reduction along the way on a few occasion). Note that we do not apply a frequency filter in our travel-time measurements, so we are not explicitly attempting to minimize these separate bands. This trend occurs organically, suggesting that the eventual result will be consistent with the governing wave equation and the measurement technique. It also adds support to the notion that the adjoint method in conjunction with linear algebraic inverse methods can be very successful. Note that we could also have used ridgeand phase-speed filtering to further test for a decreasing misfit with iteration. 

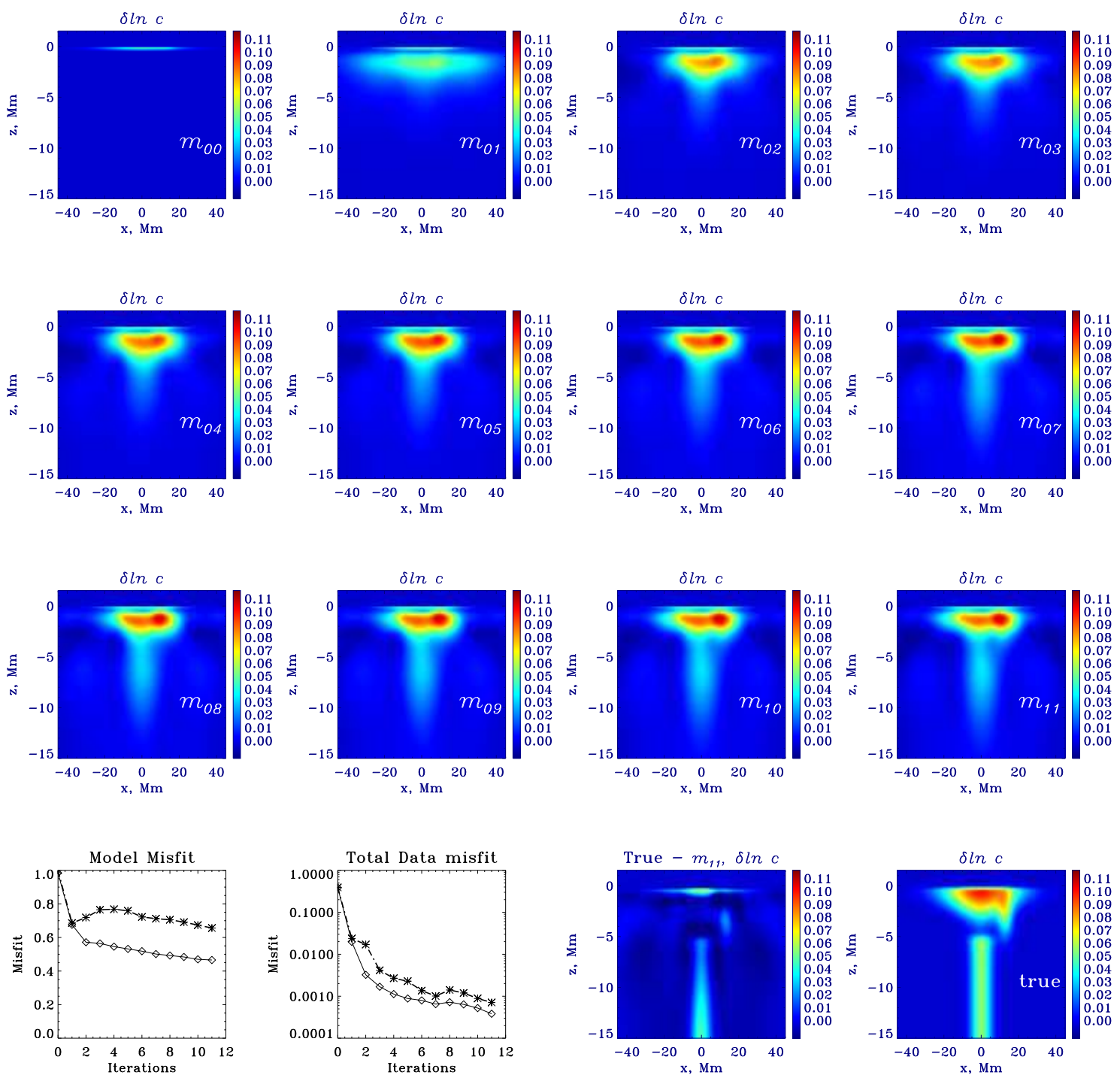

Fig. 8.- Iterations in a conjugate-gradient based inversion. The starting model contains a 'surface constraint', as seen in $m_{00}$. The rest of the algorithm is unchanged from the example shown in Figure 6. The first iteration is performed using steepest descent and a combination of conjugate gradient and steepest descent are used to compute subsequent models. It is seen that models approach the true but the reduction in the misfit slows down with iteration. The model misfit is the normalized $L_{2}$ norm difference between the true and current model whereas the total data misfit is the same as equation (11). For comparison, we over plot the misfit evolution for the unconstrained inversion (dot-dashed line with asterisks). For categories of model and data misfit, it is seen that surface constraints accelerate convergence. 

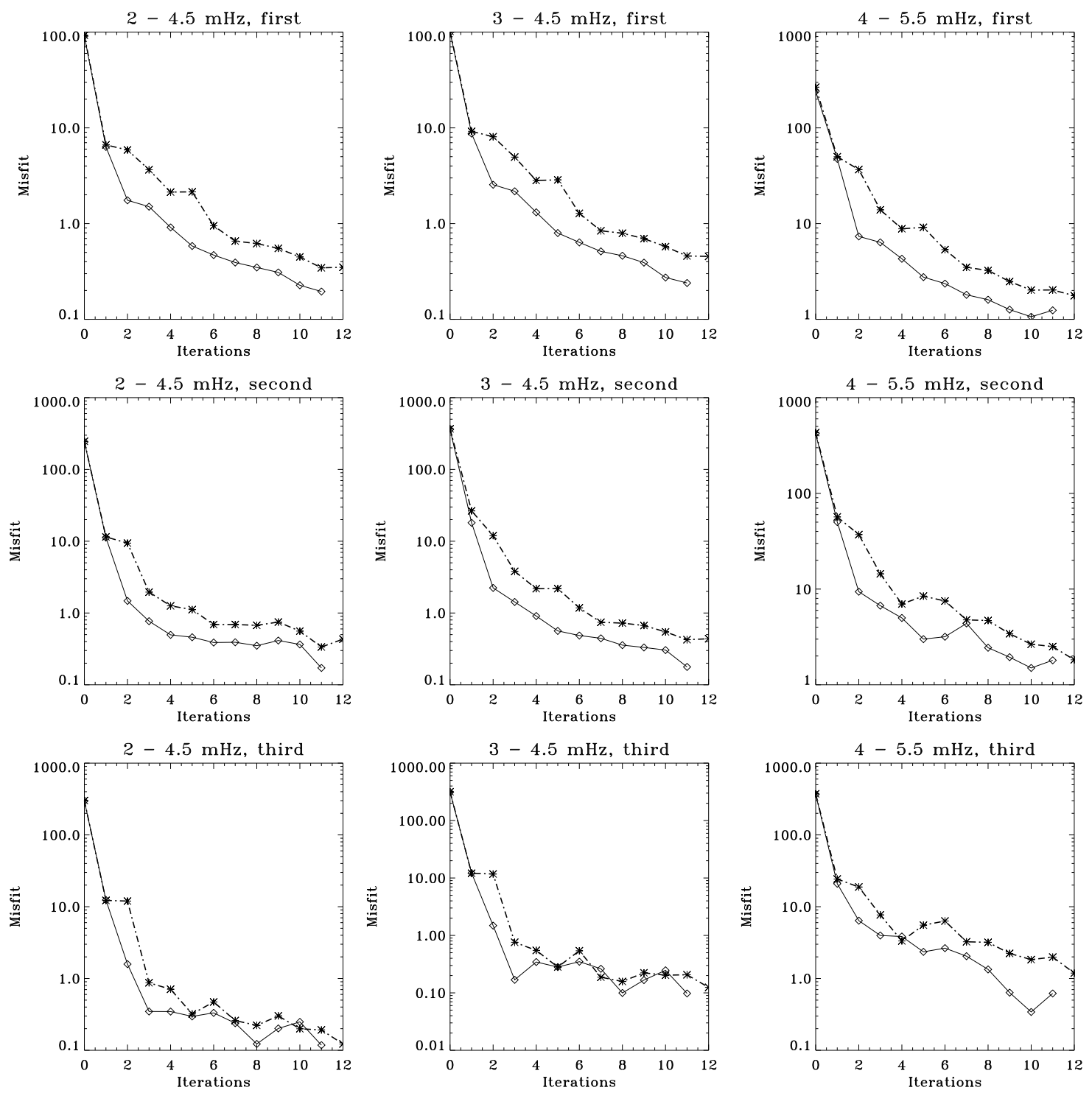

Fig. 9.- Comparison of misfit bands between surface-constrained and unconstrained inversions. Systematically, unconstrained inversions show slower convergence, as evidenced by the curves with higher misfit (dot-dashed lines with asterisk symbols). Smooth lines with circle symbols show the misfit evolving with iteration for surface-constrained inversions. 

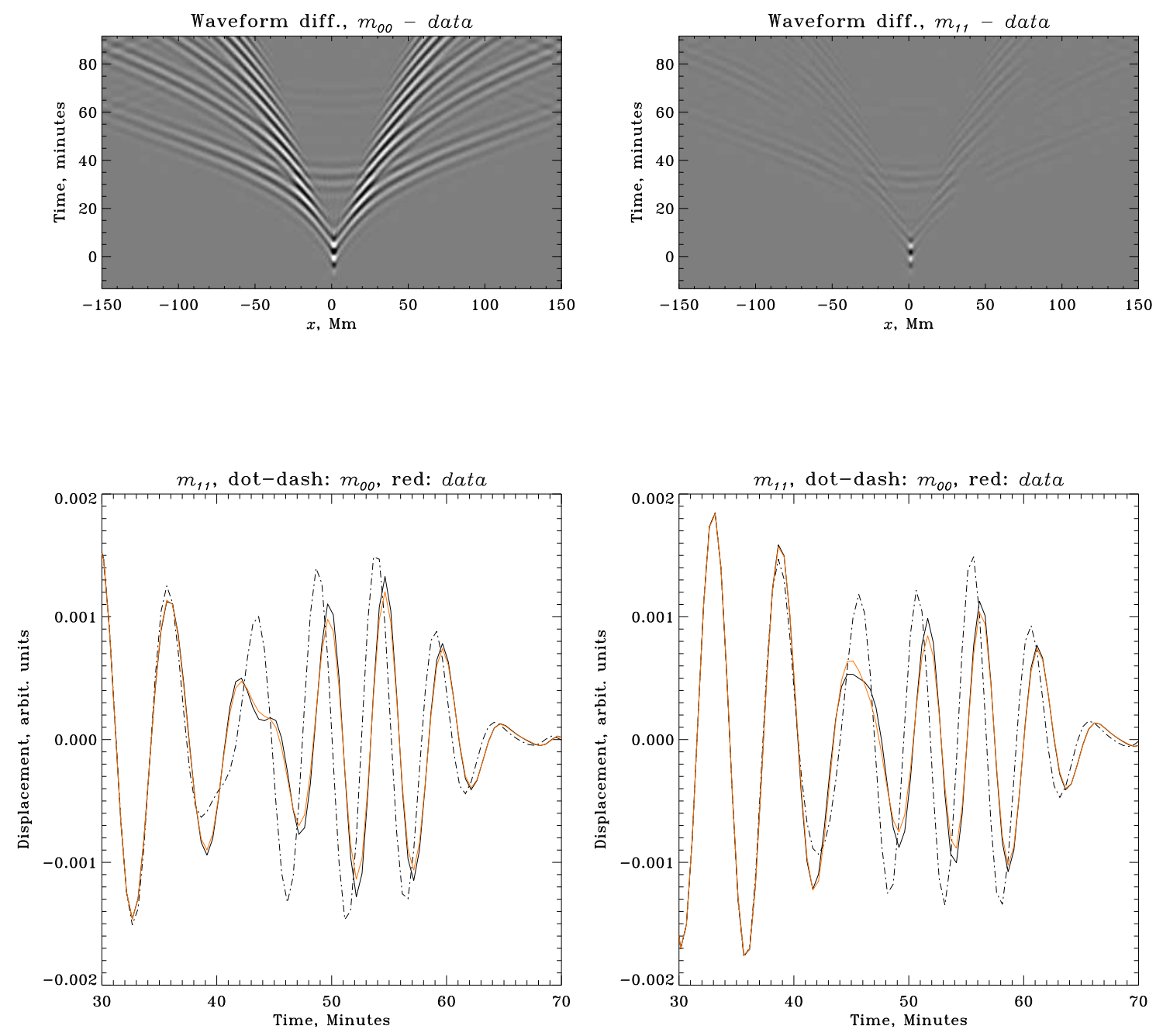

Fig. 10.- Waveform matching as a function of iteration. difference between time-distance diagrams of models $m_{00}, m_{11}$ and target data (upper panels). At iteration 11, the difference is substantially smaller (plotted on same scale). Lower panels show waveforms at $x=-9$ Mm (left) and $x=22 \mathrm{Mm}$ (right). By iteration 11, the waveforms match the data very well. 